\title{
Ensayo \\ Del comportamiento organizacional a la práctica de producción del sentido
}

\author{
Fecha de recepción: 15-01-2021 • Fecha de aceptación: 22-03-2021 • Fecha de publicación: 10-06-2021
}

\author{
Galo Hernán García Tamayo \\ Instituto Superior Tecnológico España, Ecuador \\ galoh.garciat@iste.edu.ec \\ https://orcid.org/0000-0002-2268-2730
}

\section{RESUMEN}

El presente ensayo tiene como propósito presentar algunas reflexiones sobre la práctica en la producción del sentido que se atribuyen a las organizaciones, producto del tránsito de las mismas, de la modernidad a la posmodernidad.

El trabajo consistió en hacer una revisión documental con un recorrido por la literatura respecto de la temática planteada, con el fin de entender desde un punto de vista teórico, cuáles han sido los cambios que ha soportado la sociedad desde la modernidad hasta la posmodernidad.

El estudio se basó en la lectura crítica del tema presentado; para ello se consiguieron artículos científicos, mismos que fueron analizados a profundidad con la finalidad de relevar los datos pertinentes. La comprensión de la información se complementó con métodos de análisis de contenido y de frecuencia.

Se concluye que, a lo largo de la historia, la marcada incertidumbre característica de dicha época, abre la posibilidad a una nueva vía que se dirige a la búsqueda de sentido en las personas.

\section{PALABRAS CLAVE: comportamiento organizacional, producción del sentido, organizaciones.}




\section{ABSTRACT}

The purpose of this article is to present some reflections on the practice in the production of meaning attributed to organizations, as a result of their transition from modernity to postmodernity.

The work consisted of a documentary review with a review of the literature on the subject, in order to understand from a theoretical point of view, what have been the changes that society has endured from modernity to postmodernity.

The methodology used in this work was based on the critical reading of the topic presented; for this purpose, several scientific articles were obtained, which were analyzed in depth in order to gather the pertinent data. The understanding of the information was complemented with content and frequency analysis methods.

It is concluded that, throughout history, the marked uncertainty characteristic of this era opens the possibility of a new path towards the search for meaning in people.

KEYWORDS: organizational behavior, production of sense, organizations. 


\section{Introducción}

La sociedad, a lo largo del tiempo, se ha enfrentado a grandes cambios producto de su devenir histórico, mismo que se ha derivado en una progresiva diferenciación social y distintos sentidos que han conllevado a una pregunta lógica: ¿los seres humanos están recorriendo por vías que los lleven a la producción de un sentido organizacional?

Frank López (2001), estudioso de este campo, demuestra que, bajo el enfoque tradicional, lo que se suele estudiar son las categorías del modelo analítico estructural funcionalista, así como (ayudado en la hermenéutica genealógica-fenomenológica), el comportamiento como una práctica de producción de sentido dentro del modo de vida organizacional.

Para ello, este trabajo parte con dos preguntas: ¿es el comportamiento organizacional una realidad discursiva cuyo modelo analítico lo vuelve objeto estudiable? ¿es un objetivo que preexiste a toda teoría en dónde los modelos analíticos le han ido develando su forma y dimensionamientos reales?

\subsection{Las organizaciones modernas}

Herrera (2000) manifiesta que el camino de las sociedades por distintos estadios puede ser descrito como efecto ambivalente de una creciente diferenciación global. En la misma línea, Descartes, como principal pensador de la modernidad, manifiesta que la razón es fundadora de la realidad (pienso, luego existo).

En este contexto, el sentido toma forma, ya que éste ha ido experimentando muchos cambios, que, en palabras de Rojas et al., (2001) "es cierto que la modernidad involucra las grandes modificaciones experimentadas por la humanidad, representando una importante era de trascendencia mundial" (p.26).

Pero la sociedad en su transcendencia a nivel mundial ha ido tomando ciertos rasgos que, según Bericat (2003), "el rasgo básico que caracteriza a la sociedad moderna es la creciente diferenciación de sus estructuras tanto sociales, como culturales" (p.12), entendiéndose que, dicha diferenciación tiene su entramado en la división social del trabajo y de la actividad económica en unas funciones cada vez más específicas (De Baptista, 2012, p.2).

Uno de los primeros cambios en el ámbito social y económico se dio en Inglaterra con el inicio de la incorporación de la máquina de vapor en las fábricas, en reemplazo de la mano humana, dando comienzo a la era de la revolución industrial que trajo consigo la producción a gran escala y, por ende, las relaciones de trabajo se vieron modificadas.

Adam Smith, John Stuart Mill y David Ricardo, partidarios de la economía de mercado, aproximadamente en el siglo XVII defendieron dicha economía caracterizada por la libre oferta y demanda, más, en el siglo XX, surge la revolución científica que trae consigo aportaciones sobre el modo de vida organizacional con visión hacia la productividad.

Otro de los pensadores sobre la temática planteada es Bericat (2003), quien destacó que 
la modernidad con la racionalidad específica está relacionada al vincular medios y fines. La justificación está dada por la eficiencia, esto aunado a que en la modernidad poco se admite la incertidumbre, el caos, etc.

Así mismo y de acuerdo a TWI Press (1998) el aprendizaje viene dado por modelos, métodos y técnicas que son aplicados por el gerente ante determinadas situaciones y cuya acción gerencial resulta planificada, estructurada y racional; interpretaciones de la realidad organizacional que, frente a los cambios impuestos por la globalización, se tornan insuficientes y deficientes frente a las nuevas realidades, lo cual se dificulta a medida que la organización crece (De Baptista, 2012, p.3).

La ambivalencia de la modernidad ha invitado a las organizaciones a transformarse, reinventarse, a transitar hacia un nuevo modo de vida organizacional. Como lo plantea López (2001): "entrada la segunda mitad del siglo XX se hizo ostensible una transformación aguda de las organizaciones funcionales" (p. 120), y, a decir de Senge (2005) "creo que se está formando una nueva ola: el comienzo de una época que se extenderá... y que todavía carece de nombre" (p.14).

Para otros investigadores como Loyotart, 1979; Foucault, 1994; Follari y Lanz, 1998; Derrida, 1981; Habermas, 1981; Morin \& Pakman, 1994; De Sousa, 1996; Rojas et al., 2001, se da paso a una etapa que han coincidido en llamar posmodernidad.

"Estos autores son partidarios de la importancia de la información y la comunicación, la virtualización de las organizaciones, la multidimensionalidad, la complejidad y la transversalidad como paradojas que afectan tanto a la sociedad como a las organizaciones empresariales" (De Baptista, 2012, p.3).

Unido a lo anterior, Touraine (1969), a finales de la década de los sesenta, avizoró cambios desde una sociedad de industrialización capitalista hacia una "sociedad programada" pos-industrial. Según Bell et al. (1976) y Naisbitt (1983), con la aparición de la sociedad pos - industrial surgió la primera propuesta sistematizada que tomaba en cuenta algunos factores determinantes de cambio que transcendía la lógica mecanicista, dando paso a nuevas formas de hacer negocios y de gerenciar, inspirados por las Tecnologías de la Información y Comunicación (TIC), hecho que impactó en la sociedad, en las organizaciones y el individuo (De Baptista, 2012, p.4).

\subsection{El enfoque estructural - funcional del comportamiento organizacional}

A lo largo de los años se ha podido ver que el estudio del comportamiento organizacional estuvo influenciado por los enfoques clásicos que dominaron el campo discursivo de las ciencias sociales; y es así que, al realizar un arqueo de los principales exponentes de esta corriente de pensamiento, se muestra que muchos autores sostienen un discurso sobre el comportamiento organizacional que se expresa a través de las categorías clásicas del enfoque epistemológico que se impuso con el discurso positivo.

Estos autores definen al comportamiento como la relación de unas cuantas categorías como: individuo, motivación, liderazgo, conflicto, grupo, entre otras. 
Así mismo, es importante advertir que el paradigma funcional está tan arraigado en el ámbito académico que no admite una idea distinta del comportamiento que no se exprese a través de dichos conceptos. Este paradigma funcional ha dominado tanto al ámbito académico como la estructura de los diversos libros que se producen al respecto (López, 2001).

Siguiendo en esta línea, en la primera mitad del siglo XX nació una herramienta más poderosa de la que se valió el pensamiento social, denominada el modelo analítico estructural - funcional. Este nuevo modelo concibe al comportamiento humano como la consecuencia de la interrelación funcional de determinadas estructuras o sistemas como: a) sistema cultural, b) sistema organizacional, c) sistema grupal y, d) sistema individual.

El enfoque estructural - funcional creó un campo discursivo cerrado que excluyó todo enfoque alternativo e hizo del modelo y sus categorías el comportamiento mismo, convirtiéndose en un problema para la reflexión en este campo.

Bajo la metáfora mecanicista y burocrática, De Baptista (2012) diferenció un modo de vida organizacional apoyado en la división y especialización de las tareas, organizaciones jerárquicas, piramidales y funcionales, haciendo de éstas un tejido de relaciones interpersonales que se articulan mediante las tareas especializadas.

En este modo de vida organizacional el sujeto se adaptó a una vida mucho más compleja construyendo tejidos funcionales, lo que desembocó en las funciones y tareas especializadas.

El sujeto moderno es considerado un aparato social fabricado al interior de los enmarañados tejidos organizativos de las estructuras mecanicistas, constituyendo a su vez todo un entramado organizativo, donde sólo podrá realizarse como sujeto racional, ético y epistemológico, y a cuya realización accederá mediante el despliegue práctico de sus acciones políticas, epistemológicas, económicas, sociales, morales y estéticas.

Max Weber destacó sobre la modernidad que ésta estaba alentada por la racionalidad específica, una coherencia instrumental que vinculaba medios y fines (De Baptista, 2012), ya que las organizaciones modernas se organizaban con la lógica justificada por la eficiencia.

\subsection{La práctica de producción del sentido moderno}

De acuerdo a Adam Smith, la forma organizativa de la vida laboral inglesa dominada por la organización espiritual, había cambiado tanto que el mismo Smith no tuvo más que tomar esta nueva forma organizativa como punto de partida de sus investigaciones económicas.

Esta reflexión de acuerdo a López (2001) hizo que, el análisis de la riqueza en el nuevo contexto organizacional,lo condujera a concluir que la riqueza de las naciones estaba posibilitada por una nueva manera de organización del trabajo basada en la división y especialización de las tareas.

Lo relevante de esta nueva forma de organización es que, estas formas, luego de un largo proceso político, terminaron hegemonizando los procesos de vida para luego convertirse en el ámbito 
laboral conocido posteriormente como el modo de vida moderno.

Este modelo, nacido en las filas militares, comenzaba a hegemonizar la vida fabril inglesa que más luego se convirtió en un modelo de muchas empresas a nivel mundial. Estos terminaron convertidos en matrices orgánicas a partir de las cuales, esos sujetos tendrían que producirse a sí mismos como sujetos morales, racionales y epistemológicos.

\subsection{Las organizaciones basadas en la división de las tareas y la lógica de la producción}

\section{del sentido moderno}

Para abordar este tema, es trascendente recordar que las organizaciones militares clásicas son organizaciones jerárquicas piramidales y funcionales, lo que conlleva a que este tipo de empresas se conviertan en un tejido de relaciones interpersonales articulado mediante las tareas.

En este engranaje mecánico, los sujetos se han vuelto piezas fundamentales en dónde sus cuerpos quedan descompuestos en infinitos movimientos corporales mecánicos que más tarde tomaron el nombre de therbligs (elementos básicos empleados cuando se realiza una tarea manual altamente repetitiva).

Estos therbligs harán funcionales a las tareas especializadas y a la organización funcional, logrando la eficacia y la eficiencia en su comportamiento. Estas tareas o funciones especializadas deben ser relacionadas con las categorías determinadas en los principios establecidos en la economía del movimiento.

Al relacionar estas cinco categorías con las tareas o funciones especializadas de estas organizaciones funcionales, no admiten otra modalidad de sujetos que no sean los funcionarios, los sujetos definidos y determinados por las funciones.

En este sentido, el sujeto moderno no es cualquier sujeto, sino es un artefacto social específico que se construye así mismo en la acción práctica y cognitiva de producción del sentido de su propia vida que no podrá ser sino una vida organizacional, es decir, un modo de vida organizacional.

\subsection{El sujeto en su práctica organizacional se construye así mismo como un sujeto racional}

López (2001) acertadamente al tocar este tema manifiesta que el sujeto organizado, al ir desplegando su modo de vida organizacional, va construyendo el tejido funcional que lo regula de manera estratégica, y con ellos se construye así mismo en una dialéctica organizacional donde, sin la conciencia plena de ello, termina convirtiéndose en causa de sí mismo en su propio demiurgo (p.110).

En esta lógica organizacional los sujetos sólo pueden actuar exitosamente al interior de sus 
organizaciones, sólo pueden tener un modo de vida organizacional exitoso, un comportamiento laboral deseable en la medida en que guíen sus acciones prácticas y cognitivas bajo el imperativo práctico de los fines organizacionales que son las tareas especializadas.

Los sujetos, al desplegar sus acciones prácticas y cognitivas, se incorporan a un circuito de racionalidad medio-fines, en el cual los medios terminan totalmente subordinados a los fines, lo que ha provocado un doble efecto en los sujetos modernos: los que producen en las acciones prácticas y los efectos que producen en las acciones cognitivas.

Los que producen en las acciones prácticas indican que el efecto de estas acciones se va produciendo cuando los sujetos organizados van desplegando su modo de vida organizacional. Al desplegarlo, se subordinan a los fines organizacionales y acciones prácticas, lo que les conlleva a cambiar su status como: de recibir a dar órdenes, de realizar trabajos manuales a realizar trabajos intelectuales, de recibir menores a recibir mayores remuneraciones, entre otras.

Toda esta situación sólo puede brotar de una dinámica organizacional propia de la institución militar, en dónde ese racional instrumental característico de ese tipo de instituciones se volvió la única garantía de toda victoria y de la preservación de la vida de los ejércitos.

Así mismo, Frank López (2001) sostiene que, al imponerse estos modelos organizacionales militares, esta racionalidad se erigió en lógica organizacional sobre la cual se desplegó el comportamiento mecánico al interior de las organizaciones modernas; que en el ámbito económico dio la positividad a la existencia de una fuerza productiva sobre la cual se estructuró un modelo de producción característico de la modernidad (p.112).

\subsection{La realización epistemológica o de cómo el sujeto moderno construye sus} representaciones

En este sentido, el autor López (2001) manifiesta que los juicios racionales de estos sujetos organizados terminan siendo también destrezas cognitivas, toda vez que los imperativos cognitivos son también funcionales. Pero todo ello precisamente es lo que les permite a los sujetos modernos el desarrollo de una actividad heurística que queda integrada al circuito de racionalidad y con la cual los sujetos organizados crean sus propias representaciones que le sirven de guías en el despliegue de su comportamiento organizacional y que hace exitoso su modelo de vida organizacional (p.113).

Un imperativo importante dentro de este tema radica en que la primera condición de todo enunciado verdadero, es que el sujeto que enuncia debe ser él mismo verdadero; es decir, estos sujetos van fabricando el sentido de sus propias vidas y, definiendo un comportamiento organizacional que refuerza su modo de vida moderno y el éxito de su comportamiento organizacional.

Esta relación, de acuerdo a López (2001) "será la que sostendrá el modelo de saber científico durante la modernidad clásica, que hará posible las escuelas clásicas de comportamiento, ya 
que, convertida la estructura cognitiva en una estructura funcional, todo juicio racional termina estructurándose como un juicio sintético" (p.114).

En otras palabras, todo enunciado con pretensión de verdad termina siendo un enunciado hipotético o funcional. De allí que toda verdad emergerá como hipótesis y, toda certidumbre metodológica solo podrá ser alcanzado a través de la contrastación de esas hipótesis y, mediante los procedimientos de falsación. En este aspecto López (2001) manifiesta que esto no muestra cómo el sujeto moderno, al tiempo que se fue fabricando así mismo en el entramado de un tejido orgánico funcional, se fue haciendo un funcionario racional cuya acción cognitiva, subordinada a la función, le iba permitiendo la elaboración de sus propias representaciones, con las cuáles no solo se inventaba como sujeto verdadero, sino que inventaba a su vez un discurso científico y racional sobre la economía, sobre el trabajo, sobre la administración y sobre el comportamiento organizacional (p.116).

De este modo, los sujetos al tiempo que se hacen racionales, subordinando sus acciones prácticas y cognitivas a las funciones, racionalizan sus prácticas, cumplen de manera óptima sus tareas especializadas, ascienden en la escala jerárquica de funciones organizacionales, pasando de la realización de trabajos manuales a la realización de trabajos intelectuales.

\subsection{La realización política: las positividades de los modelos de liderazgos modernos}

López (2001) manifiesta que "no obstante, en este acto único y total, dónde los sujetos se hacen racionales, virtuosos y, se realizan epistemológicamente al construir sus propias representaciones inventándose así mismo, también logran, y de una manera simultánea, su propia realización política" (p.116).

Los sujetos en la medida en que subordinan sus acciones, tanto a los fines organizacionales, como a las funciones, se convierten en funcionarios definidos por la función. De esta manera la empresa nace y va construyendo de manera progresiva una estructura política de naturaleza tecnocrática, puesto que los sujetos se convierten en la pura función técnica, volviendo racional y verdadera una estructura política caracterizada por las líneas de mando verticales sobre las cuales se sostiene el liderazgo formal y, así mismo, fue de esas estructuras de gobierno de las cuales se fueron derivando los modelos gerenciales autocráticos.

Ahora bien, en todas las organizaciones se fue dando las prácticas eleccionarias, creándose una realidad organizacional sincrética que fundía la organización disciplinaria con las formas de organización eleccionarias de dónde surgían formas de gobierno organizacionales caracterizados por las democracias centralizadas, de cuyo interior emergieron modelos gerenciales basados en el centralismo democrático, que luego fue flexibilizando la rigidez disciplinaria del gobierno organizacional autocrático, dando lugar a nuevos modelos gerenciales.

\subsection{La práctica de producción de sentido post-moderno}

En esta época hubo una transformación de las organizaciones funcionales. Es decir, se pasó de las burocracias verticales a la gran empresa horizontal, en donde se dejó la sociedad de 
empleados y se transformó en una sociedad de redes.

En este mundo post-moderno el sentido de producción ha tomado dos vías: la administrativa y la sociológica. López (2001) respecto de la administrativa manifiesta que el paso de las estructuras jerárquicas a las redes aplanadas, se observa como un proceso en el cual las funciones organizativas que caracterizaron las grandes jerarquías organizacionales, se han ido fusionando unas con otras para dar lugar a actividades, que al irse integrando entre sí han dado lugar a procesos, ya que los procesos no son más que la secuencia más o menos direccionada de un conjunto de actividades (p.122).

Respecto del área sociológica, el autor anteriormente mencionado indica que este es un proceso sociológico dado que al integrarse las funciones y construir actividades, los funcionarios que realizaban dichas funciones se integraron unos con otros formando grupos. Sin embargo, al integrarse unas actividades con otras para dar lugar a los procesos, los grupos que corresponden a esas actividades, se integraron entre sí formando equipos. (p.122).

En este sentido, el sujeto post-moderno ha entrado en un proceso en dónde ha perdido su forma disciplinaria y monológica, pero al mismo tiempo, refundándose y adoptando la forma comunicativa y simbólica.

Todo esto ha dado como resultado el nacimiento del empowerment, dejando de lado viejos modelos gerenciales centrados en la estructura.

El empowerment (empoderamiento), en palabras de López (2001), se definiría como el que moviliza a los individuos y a los equipos auto dirigidos no sólo para ejecutar órdenes, sino para innovar y mejorar los productos, los servicios y los programas, a menudo con resultados que abren nuevos caminos.

\section{Conclusiones}

En la época posmoderna se observa que se van produciendo transformaciones de interés en las estructuras organizacionales, específicamente en los modelos de gestión que fundamentan el desarrollo de las organizaciones, poniendo mayor énfasis en la necesidad de hacer reingeniería en las estructuras de organización burocrática para pasar a un siguiente nivel; es decir, a las organizaciones caracterizadas por su capacidad de evolución partiendo de la comunicación, a pesar del caos característico de esta era.

Todos estos cambios también afectan al tipo de liderazgo, en dónde el líder puede interpretar esa realidad a partir de sus principios para reconstruirla y proyectarse hacia el futuro impulsando acciones para hacerla realidad.

Por otro lado, la modernidad dio gran importancia a la racionalidad individual en dónde se visualizó cómo éstas ideas han estado presentes en la representación de las organizaciones, y, por lo tanto, en la conceptualización del liderazgo como proceso organizacional. 
Finalmente, de la revisión documental se puede concluir que la sociedad se está yendo hacia una nueva conciencia y espíritu organizacional, en dónde, la orientación está encaminada a la construcción del sentido de las personas y por ende, de las organizaciones; es decir, se vuelve importante la búsqueda de la trascendencia del ser. 


\section{Referencias}

Bell, D., García, R., \& Gallego, E. (1976). El advenimiento de la sociedad post-industrial: un intento de prognosis social. Madrid: Alianza.

Bericat, E. (2003). Fragmentos de la realidad postmoderna. Revista española de investigaciones sociológicas, 102, 9-46. http://reis.cis.es/REIS/PDF/REIS 102 031167995793345.pdf

De Baptista, O. L. S. (2012). Hacia la producción de un sentido organizacional: el dilema postmoderno. Gaceta Técnica, (9), 35-44.

De Sousa, B. (1996). Introducción a una ciencia posmoderna. (Caracas: CIPOST - FACES - UCV), Colección Estudios Avanzados 3.

DERRIDA, J. (1981). Positions. USA: University of Chicago.

Follari, R., \& Lanz, R. (1998). Enfoque sobre la postmodernidad en América Latina. Editorial Sentido. Caracas.

Foucault, M. (1994). La verdad y las formas jurídicas. Barcelona: Gedisa. Segunda Edición.

Habermas, J. (1981). Modernidad versus postmodernidad. Barcelona. En José Picó Ediciones.

Herrera, M. (2000). Representaciones de la sociedad: de la modernidad a la posmodernidad. pp. 163-190 https://ddd.uab.cat/pub/papers/02102862n61/02102862n61p163.pdf

López, F. (2001). Del comportamiento organizacional a la práctica de la producción del sentido. R. Lanz (Comp.), Organizaciones Transcomplejas, 97-144.

Loyotart, J. (1979). La Condición Posmoderna. Madrid.

Morin, E., \& Pakman, M. (1994). Introducción al pensamiento complejo. Barcelona: gedisa.

Naisbitt, J. (1983). Macrotendencias. Editorial Mitre.

Rojas, L., Torres, R., \& Arapé, E. (2001). Posmodernidad: Lógicas organizacionales, lógicas tecnológicas. Organizaciones Transcomplejas. Editorial Imposmo/Conicit, Caracas.

Senge, P. (2005). La Quinta Disciplina. Granica. Argentina.

Touraine, A. (1969). La Sociología de la Acción. Barcelona: Ediciones Ariel. 


\section{Copyright (c) 2021 Galo Hernán García Tamayo}

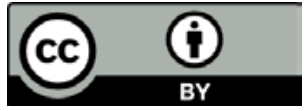

Este texto está protegido bajo una licencia internacional Creative Commons 4.0.

Usted es libre para Compartir - copiar y redistribuir el material en cualquier medio o formato - y Adaptar

el documento - remezclar, transformar y crear a partir del material-para cualquier propósito, incluso para fines comerciales, siempre que cumpla las condiciones de Atribución. Usted debe dar crédito a la obra original de manera adecuada, proporcionar un enlace a la licencia, e indicar si se han realizado cambios. Puede hacerlo en cualquier forma razonable, pero no de forma tal que sugiera que tiene el apoyo del licenciante o lo recibe por el uso que hace de la obra.

$\underline{\text { Resumen de licencia - Texto completo de la licencia }}$ 